\title{
Oocyte-specific deletion of $\mathrm{Hdac} 8$ in mice reveals stage-specific effects on fertility
}

\author{
Vijay Pratap Singh¹, Wei-Ting Yueh¹, Jennifer L Gerton ${ }^{1,2}$ and Francesca E Duncan ${ }^{3}$ \\ ${ }^{1}$ Stowers Institute for Medical Research, Kansas City, Missouri, USA, ${ }^{2}$ Department of Biochemistry and Molecular \\ Biology, University of Kansas Medical Center, Kansas City, Missouri, USA and ${ }^{3}$ Department of Obstetrics and \\ Gynecology, Feinberg School of Medicine, Northwestern University, Chicago, Illinois, USA
}

Correspondence should be addressed to J L Gerton or F E Duncan; Email: jeg@stowers.org or f-duncan@northwestern.edu

\begin{abstract}
Eighteen histone deacetylases exist in mammals. The class 1 histone deacetylases HDAC1 and HDAC2 are important for oogenesis and fertility in mice, likely via their effects on histones. The reproductive function of HDAC8, another class 1 enzyme, has not been explored. One key target of HDAC8 is the SMC3 subunit of cohesin, an essential complex mediating sister chromatid cohesion and chromosome segregation. In current models, HDAC8 activity is required for SMC3 recycling, but this function should be dispensable in oocytes since cohesion is established during pre-meiotic $S$ phase and maintained until meiotic resumption during ovulation. Whether other oocyte-specific HDAC8-mediated deacetylation events are required for oogenesis and female fertility is unknown. We used two Cre drivers to remove $\mathrm{Hdac} 8$ at specific stages of oocyte development to address whether HDAC8 is required for female fertility in mice. When HDAC8 was knocked out in oocytes in primary and later stage follicles (Zp3-Cre), oogenesis and folliculogenesis appeared normal and mice were fertile. However, females were subfertile when HDAC8 was knocked out prior to pre-meiotic S phase and cohesion establishment (Vasa-Cre). This subfertility was independent of chromosome segregation errors during meiosis but rather appeared to be the result of defects in oogenesis that resulted in smaller fully grown oocytes with a reduced ability to resume meiosis. In all cases, we did not observe compensatory changes in HDAC1, HDAC2 and HDAC3 levels. Thus, although oocyte-specific expression of HDAC8 is not essential for mouse oogenesis after meiotic $S$ phase, it contributes to optimal fertility. We infer that oocyte-specific expression of the deacetylase HDAC8 is required early in oogenesis for optimal fertility. Reproduction (2019) 157 305-316
\end{abstract}

\section{Introduction}

Oocyte quality is defined by the acquisition of both meiotic competence and cytoplasmic competence during development (Sorensen \& Wassarman 1976, Wickramasinghe et al. 1991, Wickramasinghe \& Albertini 1992). Meiotic competence refers to the ability of the oocyte to undergo meiosis to produce a euploid and haploid gamete. Cytoplasmic competence instead refers to the accumulation of key maternal factors during oogenesis, including mRNAs and proteins, which support meiotic maturation, fertilization and early preimplantation embryo development (Eppig \& Schroeder 1989). This maternal legacy is critical, since developmental events prior to the activation of the zygotic genome occur largely in the absence of transcription, and the stored cellular components of the sperm do not contribute significantly to cleavagestage embryogenesis. As a result, growing oocytes are transcriptionally and translationally active (EichenlaubRitter \& Peschke 2002, Gosden \& Lee 2010). Microarray studies have demonstrated that during oogenesis, the largest changes in oocyte gene expression occur between the primordial and primary follicle transition (Pan et al. 2005).

Critical to transcriptional regulation is modulation of chromatin accessibility through histone posttranslational modifications such as phosphorylation, methylation and acetylation (Grunstein 1997, Jenuwein \& Allis 2001, Bhaumik et al. 2007). Histone acetylation is regulated by two groups of enzymes: histone acetyltransferases (HATs) and histone deacetylases (HDACs). HATs catalyze histone acetylation, and this modification is typically associated with gene activation. On the other hand, HDACs catalyze deacetylation of histones as well as other proteins (Luo et al. 2000, Martinez-Balbas et al. 2000, Hubbert et al. 2002, Minucci \& Pelicci 2006). HDAC-mediated histone deacetylation has typically been associated with transcriptional repression, but recently it has been shown that HDACs can function in transcriptional activation as well (Greer et al. 2015). In mammals 18 HDACs have been identified, and based on their homology, they have been divided into four classes (Bolden et al. 2006). Class I HDACs (HDAC1, 2, 3 and 8) show homology with yeast Rpd3 and are expressed ubiquitously and localize to nuclei, class II 
HDACs (HDAC4, 5, 6, 7, 9 and 10) show homology to yeast Hda1 and shuttle between the nucleus and cytoplasm, class III HDACs show homology with yeast Sir2, and class IV HDACs contain only HDAC11 (de Ruijter et al. 2003).

The role of two class I HDACs (HDAC1 and HDAC2) have been extensively studied in mouse oocyte development (Ma \& Schultz 2008, 2013, Ma et al. 2012). HDAC1 and HDAC2 are concentrated in the nucleus during oocyte growth (Ma et al. 2012). Deletion of either Hdac1 or Hdac2 alone in the growing oocyte has no obvious effect on oocyte development to the fully grown stage. However, deletion of both Hdac1 and Hdac2 in the growing oocyte results in arrest of follicle development at the secondary follicle stage with an increase in global histone acetylation, increased transcription, and apoptosis, which together lead to infertility (Ma et al. 2012). These results indicate that HDAC1 and HDAC2 can compensate for each other in the oocyte. However, allelic studies indicate that HDAC2 function is more critical than HDAC1 during oocyte development (Ma \& Schultz 2016). Although studies have been performed on the two other class I HDACs (HDAC3 and HDAC8) in the oocyte, they are limited to fully grown oocytes and their roles during meiotic maturation (Zhang et al. 2017, Li et al. 2017b).

While the targets of HDAC8 in oocytes have not been established, HDAC8 has been shown generally to target non-histone proteins including the SMC3 subunit of cohesin (Durst et al. 2003, Wilson et al. 2010, Deardorff et al. 2012, Wu et al. 2013). HDAC8-mediated deacetylation of SMC3 is required for recycling of the cohesin complex so that it can be used in the next cell cycle (Beckouet et al. 2010, Borges et al. 2010, Xiong et al. 2010, Deardorff et al. 2012). Current models propose that cohesin is loaded onto chromosomes in oocytes during pre-meiotic $S$ phase and is not turned over (Revenkova et al. 2010, Tachibana-Konwalski et al. 2010, Burkhardt et al. 2016). If this is true, then recycling of SMC3 should not be necessary in oocytes, and we would predict that HDAC8 would be dispensable. However, it is possible that the deacetylation of other targets in the oocytes may be required for oogenesis. Indeed, Hdac8 knockdown or drug inhibition in fully grown oocytes was reported to cause aberrant spindles, misaligned chromosomes and aneuploidy (Zhang et al. 2017), suggesting that HDAC8-mediated deacetylation events are required for the faithful division of chromosomes and euploid eggs.

In the present study, we have examined HDAC8 function in mouse oocytes using zona pellucida spermbinding protein 3 (Zp3)-Cre(de Vries et al. 2000) and Vasa-Cre (Gallardo et al. 2007) to knockout Hdac8 (Haberland et al. 2009) at different stages of oocyte development in vivo. Our results suggest that oocytespecific HDAC8 expression is required in a stage-specific manner for optimal oogenesis and fertility. Moreover, loss of HDAC8 does not result in compensatory upregulation of other class I HDACs.

\section{Material and methods}

\section{Animals}

All mice used in this study were housed in a controlled barrier facility under constant temperature, humidity and light at the Stowers Institute for Medical Research (Kansas City, MO). Food and water were provided ad libitum. All experimental protocols were approved by the Institutional Animal Care and Use Committee of the Stowers Institute for Medical Research and were performed in accordance with guidelines and requirements.

\section{Generation of mouse lines}

Female mice carrying the Hdac8 floxed allele (fl/t), a kind gift from Eric Olson (Haberland et al. 2009) were crossed either with Zp3-Cre (JAX \#003651) (de Vries et al. 2000) or Vasa-Cre (JAX \# 006954) (Gallardo et al. 2007) male mice. Mice were genotyped as described previously (de Vries et al. 2000, Gallardo et al. 2007, Haberland et al. 2009). In the F1 generation, male mice carrying $\mathrm{Zp3}$-Cre with $\mathrm{Hdac} 8$ floxed allele (fl/0) were crossed with $\mathrm{Hdac} 8$ females (fl/ +) to generate Hdac8 $\mathrm{fl} / \mathrm{fl}$ female in a $\mathrm{Zp3}$-Cre positive or negative genetic background. Because Vasa-Cre is expressed in male germ cells, in the F1 generation, male mice carrying Vasa-Cre with Hdac8 floxed allele (fl/0) were crossed with $\mathrm{Hdac} 8$ females $(\mathrm{fl} /+)$ to generate $\mathrm{Hdac} 8 \mathrm{fl} / \mathrm{ex}$ female in a Vasa-Cre-positive or -negative genetic background. All mice with the Zp3-driven Cre expression were maintained on a $\mathrm{C} 57 \mathrm{BL} / 6$ J genetic background (JAX \#000664), whereas those with the Vasa-Cre driver were maintained on a mixed genetic background (FVB (JAX \#001800) and C57BL/6J).

\section{Oocyte isolation and Western blot analysis}

To obtain oocytes for Western blot analysis, female mice were primed with $5 \mathrm{IU}$ pregnant mare's serum gonadotropin (PMSG; RP178272, BioVendor, Brno, Czech Republic) 44 to $46 \mathrm{~h}$ prior to ovary collection. Ovaries were collected in L15 media (11415064-Life Technologies) containing $3 \mathrm{mg} / \mathrm{mL}$ polyvinylpyrrolidone (P2307-Sigma) and supplemented with $2.5 \mu$ M milrinone (M4659Sigma) to maintain meiotic arrest. Cumulus oocyte complexes were collected and oocytes were mechanically denuded of cumulus cells according to standard procedures. Sixty oocytes were pooled from mice of the same genotype and snap frozen in liquid nitrogen. Upon thawing, oocytes were lysed with Laemmli sample buffer (\#1610747-BIO-RAD), and protein extracts were separated on $4-15 \%$ Mini-PROTEAN $\left(\right.$ TGX ${ }^{\mathrm{TM}}$ Precast Protein Gels (\#4561084-BIO-RAD) and then transferred to PVDF membranes (10600029, GE Healthcare). Blots were blocked with $3 \%$ Amersham ECL prime blocking reagent (RPN418, GE Healthcare) in Tris buffered saline supplemented with $0.05 \%$ Tween (TBST) for one hour. HDAC1 (06-720, Millipore) (1:500), HDAC2 (05-814, Millipore, 05-814, Millipore) (1:500), HDAC3 (\#2632, Cell Signaling) (1:1000), HDAC8 (sc-11405, Santa Cruz) 
(1:500) and gamma tubulin (ab11316, Abcam) (1:1000) primary antibodies were diluted in blocking buffer and incubated overnight at $4{ }^{\circ} \mathrm{C}$. Blots were washed three times in TBST followed by a $1 \mathrm{~h}$ incubation in HRP-conjugated secondary antibody (NA931V and NA934V, GE Healthcare). Blots were rinsed three times in TBST and developed using the ECL ${ }^{T M}$ prime Western blotting system (RPN2232, GE Healthcare). Band intensities were quantified using ImageJ software, and HDAC levels were normalized relative to gamma tubulin.

\section{Histological analysis of ovaries and follicle counting}

Ovaries were harvested from virgin females at 18 days, 7 weeks and 7 months of age. The ovaries were fixed in Modified Davidsons fixative (64133-50-Electron Microscopy Sciences, Hatfield, PA, USA) for approximately $6 \mathrm{~h}$ at room temperature with gentle rocking before being transferred to $4^{\circ} \mathrm{C}$ overnight. After fixation, ovaries were dehydrated through an ethanol dilution series and embedded in paraffin blocks. Embedded ovaries were serially sectioned at a thickness of $5 \mu \mathrm{m}$, and sections were mounted on positively charged slides. Sections were stained with either hematoxylin and eosin (H\&E) or periodic acid-Schiff (PAS; Surgipath Schiff Reagent, Leica Biosystems. \#3803800) using the ST Infinity H\&E staining system (Leica Biosystems) following standard protocols.

Stained slides were scanned using an Olympus VS120 with a $40 \times$ objective. Morphologically normal follicles were counted in every fifth section through the entire ovary from each mouse as described previously (Bristol-Gould et al. 2006). Follicle stages were classified according to morphological criteria (Duncan et al. 2017). In brief, primordial follicles were classified as an oocyte surrounded by squamous cells, primary follicles were classified as oocytes surrounded by single layer of cuboidal granulosa cells, secondary follicles were classified as oocytes surrounded by more than one layer of granulosa cells, early antral follicles showed a visible cavity and large antral follicle contained a large antral cavity. For secondary to large antral follicles, we only counted follicles with a visible nucleus to avoid counting a follicle more than once. Total counts for each follicle class were divided by the total number of sections that were counted, and the data are represented as the average number of follicles per section.

\section{Fertility and litter size analysis}

To analyze the fertility of female mice with various genotypes, mutant female mice (6-8 weeks old) were bred with wild-type C57BL/6J mice. Mice were mated continuously for 6 months and litter number and size were monitored. The number of pups born on the first day after parturition was reported as the litter size. To calculate the average litter size, the total number of pups produced during the breeding trial was divided by total number of litters.

\section{In vitro maturation}

For in vitro maturation, germinal vesicle-intact oocytes arrested in prophase of meiosis I were collected in M2 medium (M7167-Sigma) supplemented with $2.5 \mu \mathrm{M}$ milrinone to maintain meiotic arrest. Phase contrast images of oocytes were captured using a Zeiss Axiovert 200M microscope. After imaging, oocytes were washed with M16 medium (M7292Sigma) four times, then incubated in $5 \% \mathrm{CO}_{2}$ incubator at $37^{\circ} \mathrm{C}$ for $8 \mathrm{~h}$ in $\mathrm{M} 16$ medium to mature to metaphase of meiosis I (MI) (Zhang et al. 2017). Oocytes were then fixed in $3.8 \%$ paraformaldehyde in $1 \mathrm{X}$ PBS containing $0.1 \%$ Triton $\mathrm{X}-100$ for $1 \mathrm{~h}$ at $37^{\circ} \mathrm{C}$. Oocytes diameter was measured using Image software. Diameter was determined by taking the mean of two perpendicular measurements from plasma membrane to plasma membrane..

\section{Immunofluorescence of oocytes}

For immunofluorescence, fixed oocytes were washed two times with blocking buffer (1X PBS, 0.3\% BSA, 0.01\% Tween20 and $0.02 \% \mathrm{NaN}_{3}$ ), then permeabilized for $20 \mathrm{~min}$ (1X PBS, $0.3 \%$ BSA, $0.1 \%$ Triton $\mathrm{X}-100$ and $0.02 \% \mathrm{NaN}_{3}$ ) at room temperature. Oocytes were then washed twice with blocking buffer and incubated in an anti $\alpha$-tubulin antibody (T6199Sigma) (1:500 dilution) in blocking buffer containing 5\% goat serum (S-1000, Vector Laboratories, Burlingame, CA, USA), overnight at $4^{\circ} \mathrm{C}$. The next morning oocytes were washed three times and incubated with Alexa Fluor-488 secondary antibody (A-11017-Life Technologies) (1:500 dilution) for $1 \mathrm{~h}$ at room temperature in blocking buffer containing $5 \%$ goat serum. Finally, oocytes were washed three times and mounted on slides in mounting medium containing 4',6-diamidino2-phenylindole, dihydrochloride (DAPI) (H-1200, Vector Laboratories). Oocytes were imaged using a LSM780 confocal microscope (Zeiss), and $0.3 \mu \mathrm{m}$ optical sections were taken. Z-stacks were generated, and the number of oocytes that reached $\mathrm{MI}$ were scored based on spindle and chromosome morphology. In cells at $\mathrm{MI}$, we also analyzed whether chromosomes were aligned on the metaphase plate.

\section{Chromosome spreads}

Chromosome spreads were prepared as described previously (Hodges \& Hunt 2002). In brief, mice were injected with 5 IU PMSG and oocytes were collected in L15/PVP medium containing $2.5 \mu \mathrm{M}$ milrinone, $48 \mathrm{~h}$ post injection. Oocytes were washed and cultured in M16 medium for $14 \mathrm{~h}$ and treated with Tyrode's solution $\mathrm{pH} 2.5$ to remove the zona pellucida. Oocytes were washed with M2 medium and then placed into a $\sim 60 \mu \mathrm{L}$ drop of $1 \%$ PFA in distilled water containing $3 \mathrm{mM}$ dithiothreitol and $0.1 \%$ Triton $\mathrm{X}-100$ on a clean microscope slide. After $2 \mathrm{~h}$ in a humid chamber, the slides were dried and then stored at $-20^{\circ} \mathrm{C}$. Immunofluorescence was performed as described in previous section using anti-CREST antibody (1:100, HCT-0100, ImmunoVision) to stain centromeres.

\section{In vitro zygote culture}

For zygote culture, mice were superovulated following standard hyperstimulation protocols using 5IU PMSG followed by injection of $5 \mathrm{IU}$ human chorionic gonadotropin (hCG) 46-48 h later. Mice were then mated with wild-type males, and zygotes were collected into M2 medium from plugged 
females. After collection, fertilized zygotes were confirmed by the presence of pronuclei, and they were then rinsed and cultured in $\mathrm{KSOM}$ media (MR-106-D, Millipore) at $37^{\circ} \mathrm{C}$ in a humidified atmosphere of $5 \% \mathrm{CO}_{2}$ in air. Every day the medium was changed, and images were recorded to count the number of zygotes progressing to the 2-cell, 4-cell, morula and blastocyst stages.

\section{Statistics}

Ratio values were calculated using Microsoft Excel 2016. Statistics were analyzed and graphs were generated using GraphPad Prism software (GraphPad Software). The student t-test or chi-squared test was used to determine statistical significance. A $P$ value less than 0.05 was considered significant.

\section{Results}

\section{Expression of class I HDACs in HDAC8 knockout oocytes}

To understand the role of HDAC8 in oocyte development, we obtained a conditional allele of Hdac 8 where exon 4 was flanked with loxP sites (Haberland et al. 2009). We knocked out Hdac8 specifically in early growing oocytes by using the zona pellucida 3 (Zp3)-Cre transgenic line (deVries et al. 2000). Mice bearing this transgene express Cre recombinase under the control of the $Z p 3$ promoter which is active in oocytes of primary and later stage follicles (Lan et al. 2004, Cordeiro et al. 2015). Of note, cohesion and SMC3 association with chromosomes is already established at this time. To confirm that HDAC8 was deleted in conditional knockout animals, we isolated fully grown oocytes from Hdac $8^{\text {(floxed/floxed) }}$ and Zp3Cre: $H d a c 8^{\text {(floxed/floxed) }}$ mice and performed immunoblot analysis on protein extracts (Fig. 1). Oocytes from Zp3cre: $H d a c 8^{\text {(floxed/floxed) }}$ mice showed an approximate $80 \%$ reduction in HDAC8 protein levels relative to controls (Fig. 1A and B). To further confirm this knockdown, we performed immunofluorescence with two different antiHDAC8 antibodies: Santa Cruz; sc-11405 and Abcam; ab187139. However, with both of these antibodies we observed a similar pattern of immunofluorescence in both Hdac8 (floxed/floxed) and Zp3-Cre: Hdac ${ }^{\text {(floxed/floxed) }}$ oocytes (Supplementary Fig. 1, see section on supplementary data given at the end of this article). The immunofluorescence signals with both these antibodies are likely non-specific, since both antibodies recognize multiple bands on Western blots (Fig. 1A and C; Zhang et al. 2017). These results, therefore, preclude the use of either these antibodies for immunofluorescence. Moreover, they raise the possibility that the previously reported HDAC8 colocalization with gamma tubulin in oocytes at metaphase of meiosis I may be non-specific since the same Abcam; ab187139 antibody was used (Zhang et al. 2017).

In previous studies, compound knockout of Hdac1 and Hdac2, two other class I HDAC family members,
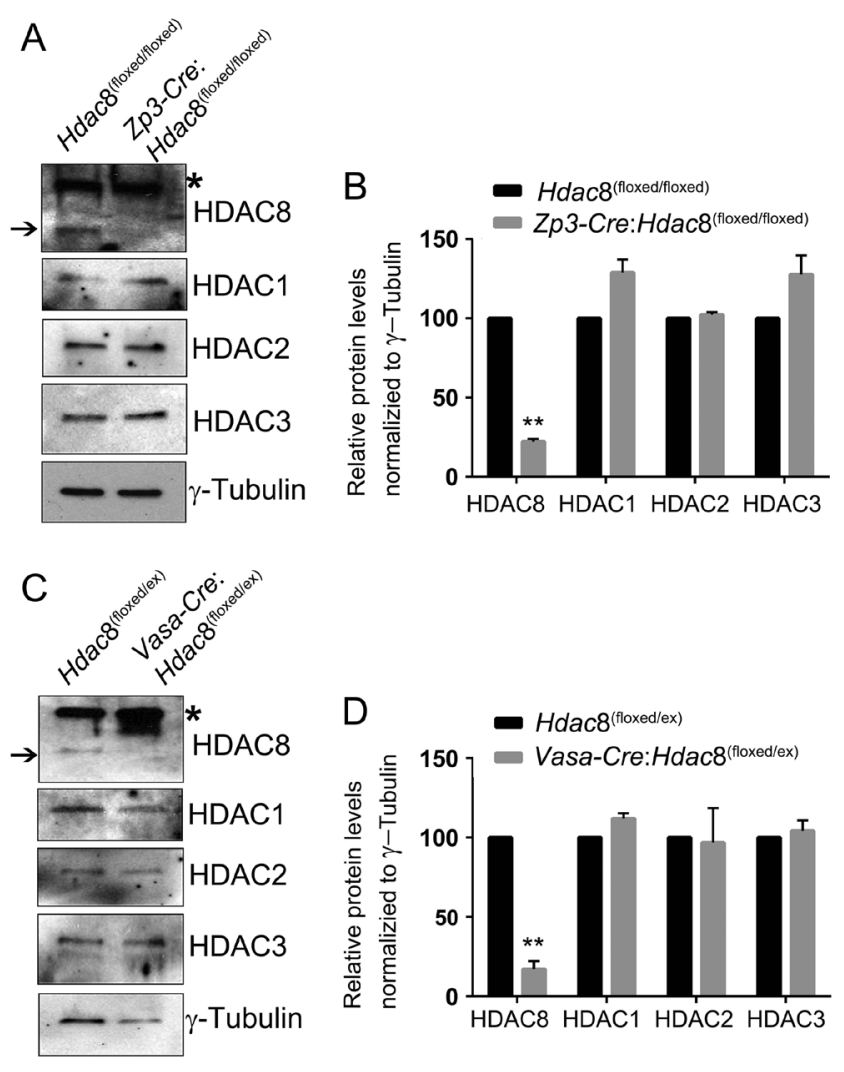

Figure 1 HDAC8 levels in the oocyte are reduced in conditional knockout animals without a compensatory upregulation of HDAC1-3. (A) Immunoblot analysis of HDACs in oocyte protein extracts from Zp3-Cre:Hdac $8^{\text {(floxed/floxed) }}$ and $\mathrm{Hdac} 8^{\text {(floxed/floxed) }}$ mice. (B) Densitometry analysis of HDAC protein levels were performed and values were normalized to $\gamma$-tubulin levels. Each experiment was performed in duplicate. (C) Immunoblot analysis of HDACs in oocyte protein extracts from Vasa-Cre:Hdac $8^{\text {(floxed/ex) }}$ and $H d a c 8^{\text {(floxed/ex) }}$ mice. (D) Densitometry analysis of HDAC protein levels were performed and values were normalized to $\gamma$-tubulin levels. Each experiment was performed in duplicate. The arrow highlights the HDAC8

immunoreactive band, whereas the asterisks highlight a non- specific band. Data are expressed as mean \pm S.E.M. $(* P<0.05)$.

resulted in a $50 \%$ reduction of HDAC8 protein levels (Ma et al. 2012). To determine whether loss of oocytespecific HDAC8 impacted the expression of the other class I HDACs, we performed immunoblot analysis for HDAC 1, 2 and 3. We found that levels of these HDACs were unchanged between oocytes from $H d a c 8^{\text {(floxed/floxed) }}$ and Zp3-Cre: Hdac8 $8^{\text {(floxed/floxed) }}$ mice (Fig. $1 \mathrm{~A}$ and B). In addition to the Zp3-Cre driver, we also generated conditional knockouts using the Vasa-Cre transgene, which is expressed at the beginning of gonad colonization by primordial germ cells, allowing for knockout of Hdac8 very early in oocyte development prior to pre-meiotic $\mathrm{S}$ phase and cohesion establishment (Gallardo et al. 2007). With this driver, we observed an $83 \%$ reduction in HDAC8 protein levels in fully grown oocytes derived from Vasa-Cre: $\mathrm{Hdac}^{\text {(floxed/ex) }}$ relative to Hdac $8^{\text {(floxed/ex) }}$ mice (Fig. 1C and D). We also examined 
the expression of other class I HDACs and found that their levels remained constant between oocytes from both $\mathrm{Hdac} 8^{\text {(floxed/ex) }}$ and Vasa-Cre: Hdac $8^{\text {(floxed/ex) }}$ mice (Fig. 1C and D). Taken together, these results suggest that both Zp3 and Vasa-Cre drivers can be used to effectively knockout HDAC8 in early stages of oocyte development without compensatory changes in expression of the other class I HDACs.

\section{Follicular dynamics in Zp3-Cre-mediated loss of HDAC8}

After confirming the reduction of HDAC8 in oocytes using the Zp3-Cre driver, we next analyzed whether there was an effect on reproductive function. We first examined folliculogenesis by analyzing ovarian histology at different ages (Fig. 2). We classified and counted follicles (primordial, primary, secondary, early antral and large antral follicles) according to established morphological criteria (Duncan et al. 2017). In prepubertal mice (18 days old), ovaries from $H d a c 8^{\text {(floxed/floxed) }}$ and Zp3-Cre:Hdac8 (floxed/floxed) mice showed similar histology with predominantly primordial, primary and secondary follicles present within the tissue (Fig. 2A). Follicle counts corroborated these observations as there were no differences in follicle number between the genotypes (Fig. 2B). In reproductively young adult mice (7 weeks old), there were also no significant differences in follicle counts between $H d a c 8^{\text {(floxed/floxed) }}$ and Zp3-Cre:Hdac8 $8^{\text {(floxed/floxed) }}$ mice (Fig. 2C and D). Importantly, we observed large antral follicles and corpora lutea in Zp3-Cre:Hdac8 $8^{\text {(floxedfloxed) }}$ mice, suggesting that the animals were cycling and able to ovulate (Fig. 2C). To examine whether loss of HDAC8
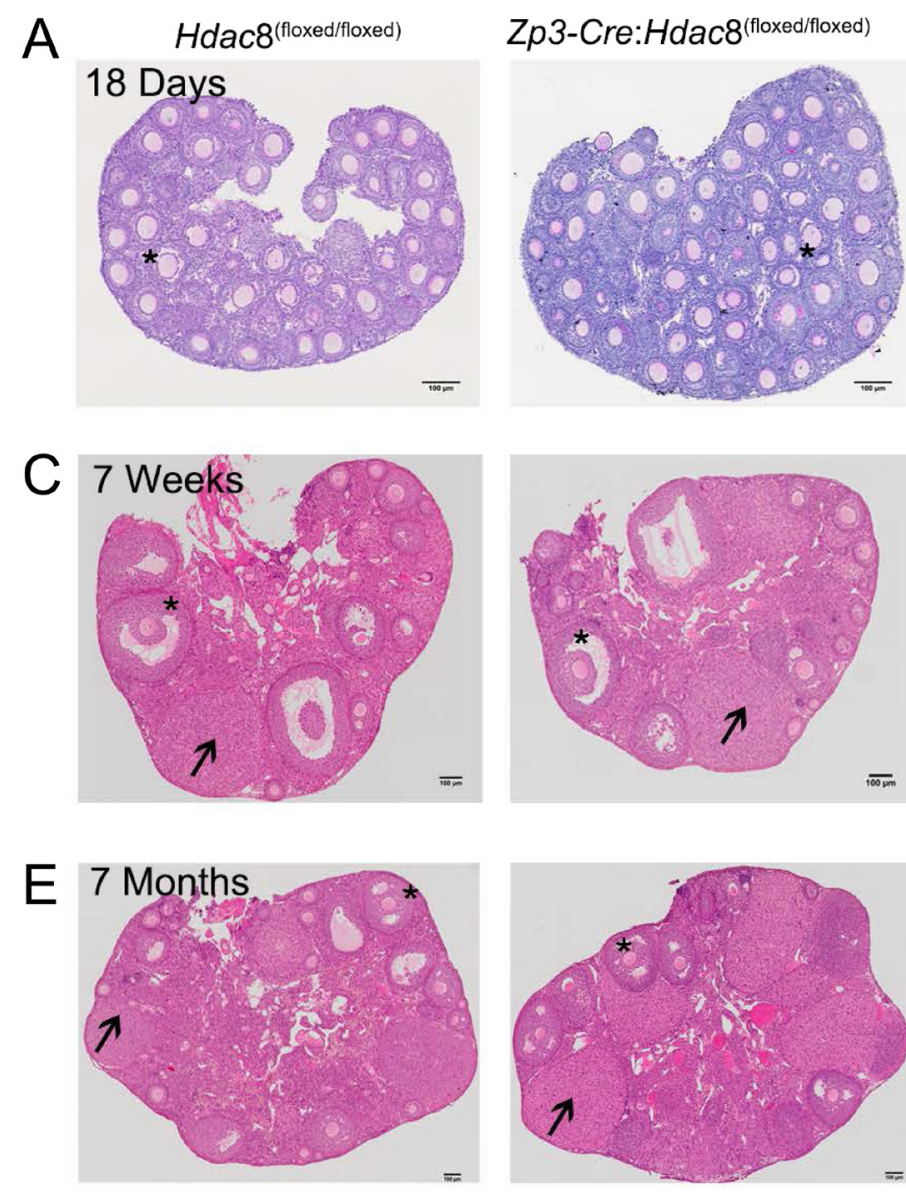
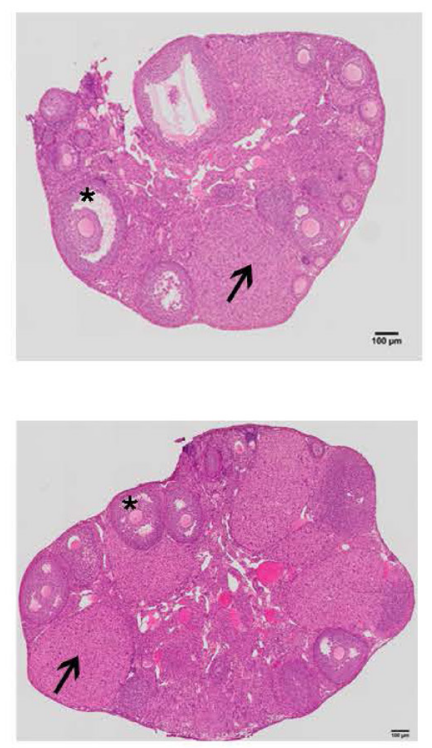
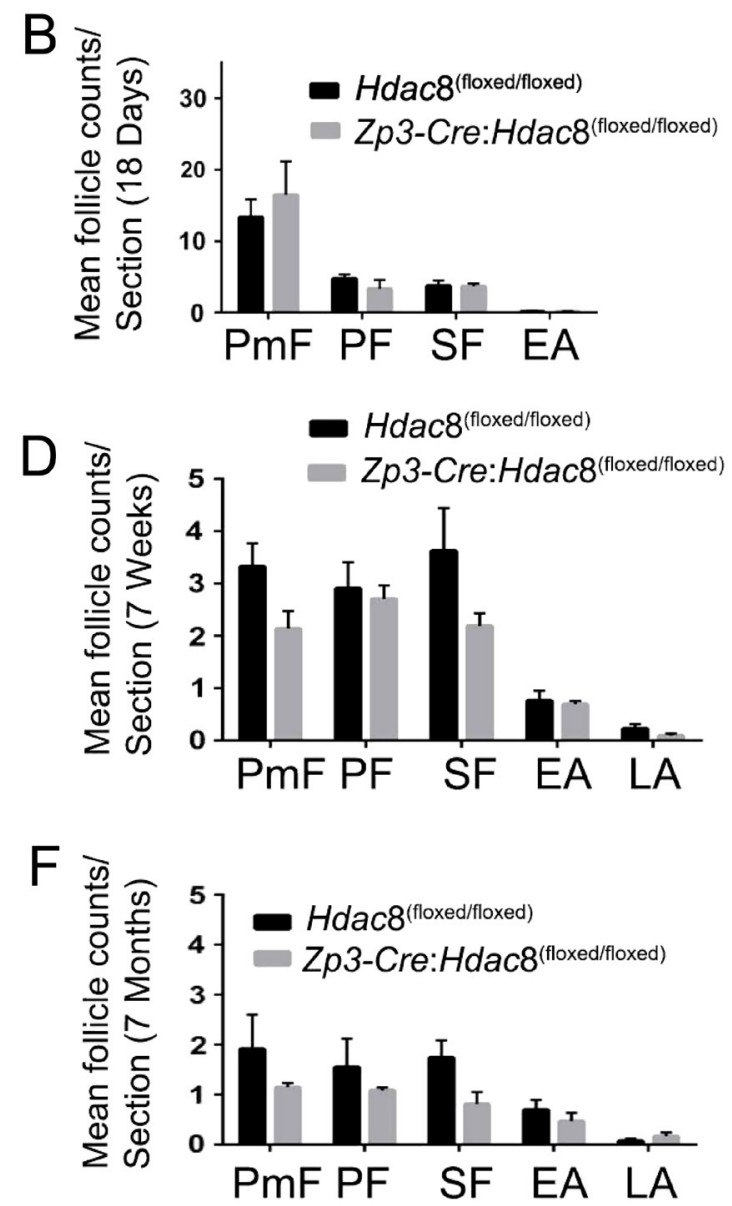

Figure 2 Zp3-Cre-mediated loss of oocyte-specific HDAC8 does not affect follicular dynamics. (A) Histologic sections of ovaries stained with periodic acid-Schiff (PAS) from Zp3-Cre:Hdac $8^{\text {(floxed/floxed) }}$ and $H d a c 8^{\text {(floxed/floxed) }}$ mice at 18 days of age. (B) Follicle quantification in ovaries from 18-day old mice ( $n=3$ mice from each genotype). (C) Hematoxylin and Eosin (H\&E) stained histologic sections of ovaries from 7-week-old mice of each genotype. (D) Follicle quantification in ovaries from 7 -week-old mice ( $n=3$ mice from each genotype). (E) H\&E stained histologic sections of ovaries from 7-month-old mice of each genotype. (F) Follicle quantification in ovaries from 7-month-old mice $(n=3$ mice from each genotype). A student $t$-test was performed to examine statistical significance between genotypes at each stage of follicle development. No statistically significant differences were observed. Data are expressed as mean \pm S.E.M. $\left({ }^{*} P<0.05\right)$. The asterisks highlight follicles with antral cavities, and the arrows highlight corpora lutea. EA, early antral follicle; LA, large antral follicle; PF, primary follicle; PmF, primordial follicle; SF, secondary follicle. 
could accelerate reproductive aging, we analyzed ovaries from 7-month-old mice (Fig. 2E). At this age, Zp3-Cre: Hdac8 $8^{\text {(floxed/floxed) }}$ mice had similar histology to $H d a c 8^{\text {(floxed/floxed) }}$ mice, and in both cases, large antral follicles and corpora lutea were visible (Fig. 2E). Follicle counting showed a similar number of follicles at all stages of development in aged Hdac $8^{\text {(floxed/floxed) }}$ and Zp3-Cre: Hdac8 $8^{\text {(floxed/floxed) }}$ mice (Fig. 2F). Taken together, these results suggest that oocyte-specific loss of HDAC8 beginning in the early growing follicle stage does not affect ovarian development or follicle numbers.

\section{Follicular dynamics in Vasa-Cre-mediated loss of HDAC 8}

Since we did not observe a phenotype with oocytespecific loss of HDAC8 when we used the Zp3-Cre driver, we analyzed ovarian histology and follicle development using Vasa-Cre-mediated loss of HDAC8. This driver expresses $\mathrm{Cre}$ in the oocyte earlier in development compared to $\mathrm{Zp3}-\mathrm{Cre}$, and is active in oocytes during meiotic prophase I and within primordial follicles (Gallardo et al. 2007). Ovarian histology between $H d a c 8^{\text {(floxed/ex) }}$ and Vasa-Cre: Hdac $8^{\text {(floxed/ex) }}$ mice were indistinguishable both at 18 days and 7 months of age (Fig. 3A and C). Follicle quantification demonstrated that there were no differences in the number of follicle classes between genotypes in both age cohorts (Fig. 3B and D). Furthermore, large antral follicles and corpora lutea were visible in ovaries from Vasa-Cre: $H d a c 8^{\text {(floxed/ex) }}$ mice, suggesting that the reproductively adult mice are cycling and able to ovulate (Fig. $3 \mathrm{C}$ and D). These results indicate that oocyte-specific loss of HDAC8 even early in development does not affect ovarian development, follicle formation, or growth dynamics.

\section{Fertility of oocyte-specific HDAC8 knockout mice}

Although we did not observe overt phenotypes of oocyte-specific loss of HDAC8 on folliculogenesis, we wanted to examine whether there were any alterations in oogenesis that would compromise oocyte quality. We, therefore, performed fertility trials to assess the functionality of HDAC8 null oocytes. We bred reproductively adult Zp3-Cre: $\mathrm{Hdac}^{\text {(floxed/floxed) }}$ female mice with wild type male mice in a standard six month breeding trial. During this time, $\mathrm{Zp3}-\mathrm{Cre}: \mathrm{Hdac}^{\text {(floxed/floxed) }}$ and $H d a c B^{\text {(floxed/floxed) }}$ mice produced a total of 5 litters, and litter sizes were similar across genotypes (Fig. 4A). In addition, the litter size was constant across the trial, suggesting that there were not age-associated changes in fertility (Fig. 4A). Total litter size during this breeding
A

$H_{d a c 8} 8^{(f l o x e d / e x)}$

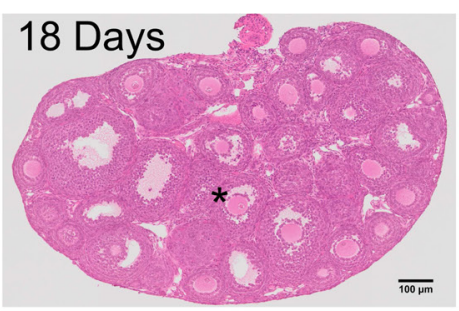

C

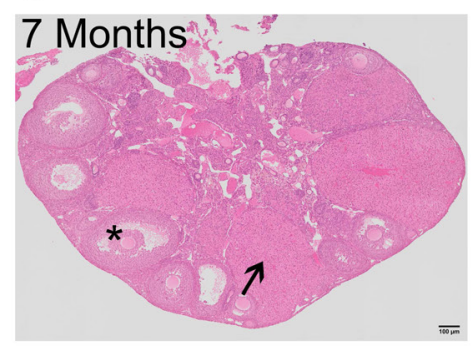

Vasa-Cre:Hdac8 ${ }^{\text {(lloxed/ex) }}$
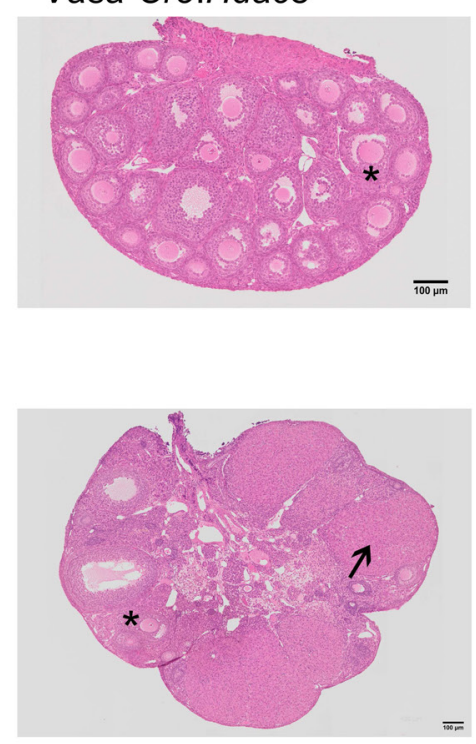

B
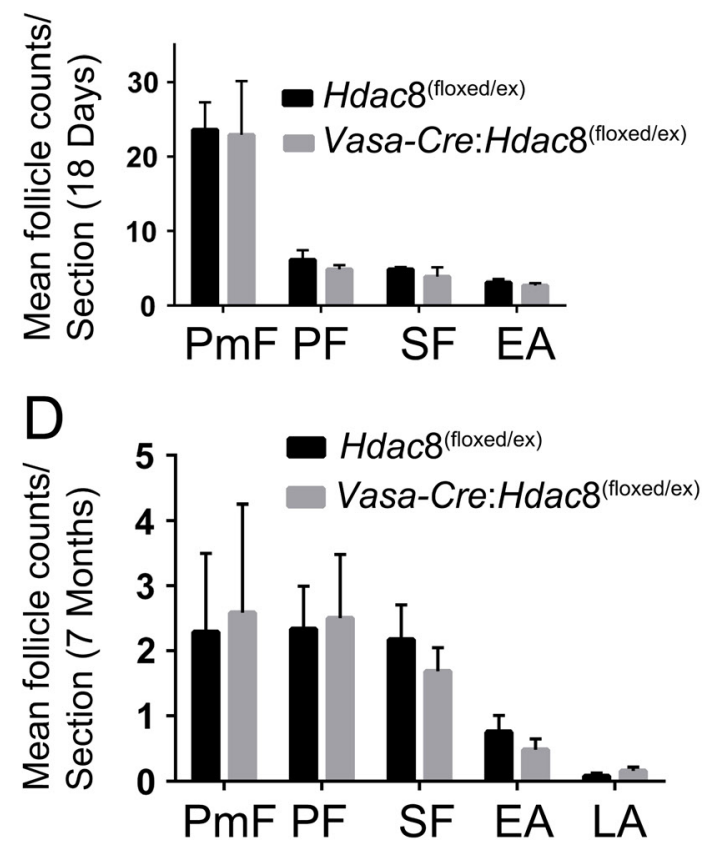

Figure 3 Vasa-Cre-mediated loss of oocyte-specific HDAC8 does not affect follicular dynamics. (A) H\&E stained histologic sections of ovaries from Vasa-Cre:Hdac ${ }^{\text {(floxed/ex) }}$ and $H d a c 8^{\text {(floxed/ex) }}$ mice at 18 days of age. (B) Follicle quantification in ovaries from 18-day-old mice ( $n=3$ mice from each genotype). (C) H\&E stained histologic sections of ovaries from 7-month-old mice of each genotype. (D) Follicle quantification in ovaries from 7-month-old mice ( $n=3$ mice from each genotype). A student $t$-test was performed to examine statistical significance between genotypes at each stage of follicle development. No statistically significant differences were observed. Data are expressed as mean \pm S.E.M. $\left({ }^{*} P<0.05\right)$. The asterisks highlight follicles with antral cavities, and the arrows highlight corpora lutea. EA, early antral follicle; LA, large antral follicle; PF, primary follicle; PmF, primordial follicle; SF, secondary follicle. 

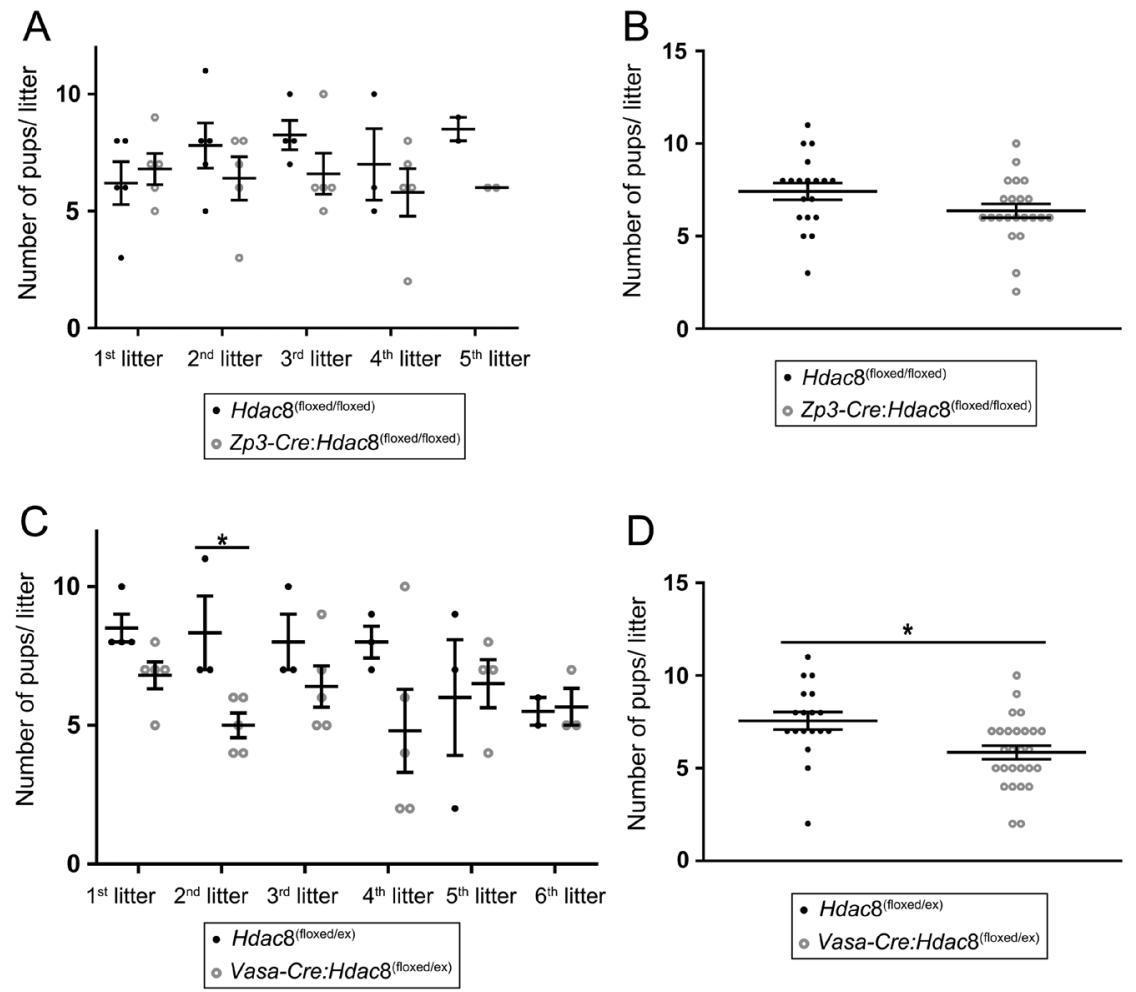

Figure 4 Oocyte-specific loss of $\mathrm{Hdac} 8$ results in subfertility in a stage-specific manner. (A) Zp3-cre-mediated deletion of $\mathrm{Hdac8}$ did not affect the number or size of litters in a standard 6 months breeding trial $(n=5$ mice for both the Zp3-Cre:Hdac8 $8^{\text {(floxed/floxed }}$ and $H d a c 8^{\text {(floxed/floxed) }}$ genotypes). Each point on the graph represents the litter size from an individual female; females produced different numbers of litters. (B) Zp3-cre-mediated deletion of $\mathrm{Hdac} 8$ does not affect the average litter size from all the females in a standard 6 months breeding trial. (C) Mice with the Vasa-cre-mediated deletion of Hdac8 had a tendency to have smaller litter sizes in the first 4 litters in a standard 6 months breeding trial, and this was significant for the second litter ( $n=5$ mice for the Vasa-Cre:Hdac $8^{\text {(floxed/ex) }}$ genotype and $n=4$ mice for the Hdac $8^{\text {(floxed/ex) }}$ genotype). Each point on the graph represents the litter size from an individual female; females produced different numbers of litters. (D) Vasa-cre-mediated deletion of Hdac8 showed reduced average litter size from all the females in a standard 6 months breeding trial. A student $t$-test was performed to examine statistical significance between genotypes and data are expressed as mean \pm S.E.M. $(* P<0.05)$. trial was similar in Zp3-Cre: Hdac8 $8^{\text {(floxed/floxed) }}$ and $H_{d a c} 8^{\text {(floxed/floxed) }}$ mice (Fig. 4B). We also performed a similar breeding trial with the Vasa-Cre: $H_{d a c} 8^{\text {(floxed/ex) }}$ mice in which reproductively adult females were bred with wild-type males. During a 6 month period, Vasa-Cre:Hdac8 $8^{\text {(floxed/ex) }}$ and Hdac ${ }^{\text {(floxed/ex) }}$ mice produced a total of 6 litters (Fig. 4C). Although the early litter sizes were smaller for the mutant, this effect diminished by the 5th and 6th litter as the wild-type litter size diminished. Taken together, the average litter size from Vasa-Cre: $H d a c 8^{\text {(floxed/ex) }}$ mice was significantly lower than control (Fig. 4D). These results indicate that loss of Hdac8 expression in primary and later stage oocytes is dispensable for female fertility. However, early loss of Hdac8 prior to pre-meiotic S phase and cohesion establishment was associated with subfertility.

\section{Meiotic competence of HDAC8 knockout oocytes}

To investigate the potential mechanism of subfertility observed with the Vasa-Cre driver, we examined the ability of the HDAC8 knockout oocytes to undergo meiotic maturation because a previous study demonstrated that acute RNAi-mediated knockdown of HDAC8 results in oocytes with meiotic defects at metaphase of meiosis I (MI) (Zhang et al. 2017). Therefore, we collected fully grown oocytes from hyperstimulated female mice of each genotype and performed in vitro maturation (IVM) (Fig. 5). There was no significant difference in the number of oocytes collected between knockout and wild-type genotypes. For example, 21.0 \pm 2.6 oocytes/ mouse were collected in the Vasa-Cre:Hdac $8^{\text {(floxed/ex) }}$ genotype compared to $26.7 \pm 6.0$ oocytes/mouse in the $H_{d a c} 8^{\text {(floxed/ex) }}$ genotype. In the Zp3-Cre:Hdac $8^{\text {(floxed/floxed) }}$ mice, an average of $23.0 \pm 3.5$ oocytes were collected/ mouse compared to $28.2 \pm 4.6$ oocytes/mouse in the Hdac $8^{\text {(floxed/floxed) }}$ genotype. In addition, oocytes from both mutant genotypes had normal nuclear and nucleolar morphology for cells arrested in prophase of meiosis I (Fig. 5A and B). Interestingly, oocytes from the Vasa-Cre:Hdac $8^{\text {(floxed/ex) }}$ mice were significantly smaller ( $n=63$ ) compared to oocytes from the Hdac $8^{\text {(floxed/ex) }}$ mice $(n=80)$ (Fig. 5B and C). No difference in oocyte diameter was observed with the Zp3-Cre driver (Fig. 5A and C). To examine the meiotic competence of these oocytes, we performed IVM and scored the ability of the cells to resume meiosis and reach $\mathrm{Ml}$ within $8 \mathrm{~h}$. Progression to the MI stage was approximately $90 \%$ in all genotypes except in oocytes from the Vasa-Cre:Hdac $8^{\text {(floxed/ex) }}$ where only $80 \%$ reached $\mathrm{MI}$, a statistically significant reduction (Table 1 ).

Because it was previously reported that RNAi-mediated depletion of HDAC8 resulted in spindle assembly defects (Zhang et al. 2017), we analyzed spindle morphology at the $\mathrm{MI}$ stage following IVM in mutant backgrounds (Fig. 6A, B, C and D). Spindle morphology was similar across genotypes, with bipolar spindles containing chromosomes tightly aligned on the metaphase plate observed equivalently in oocytes from Hdac $8^{\text {(floxed/floxed })}$ $(96.5 \%, n=68)$, Zp3-Cre: Hdac ${ }^{\text {(floxed/floxed) }}(96.8 \%, n=31)$, 
A

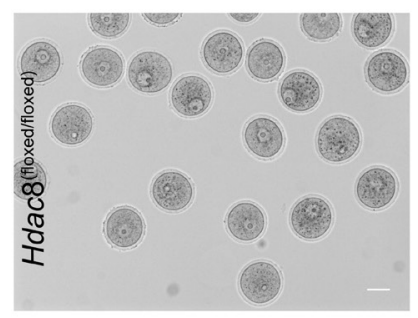

B
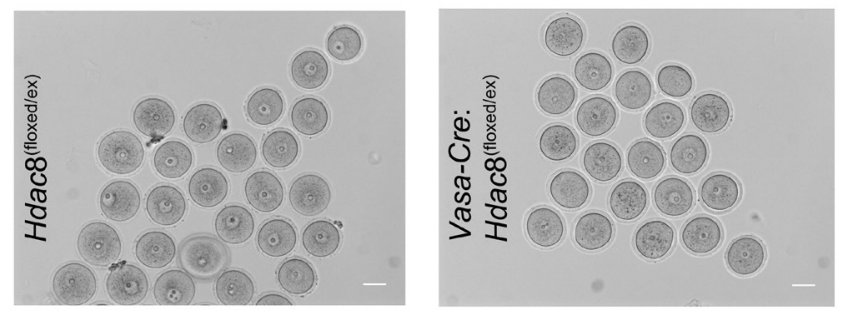

C

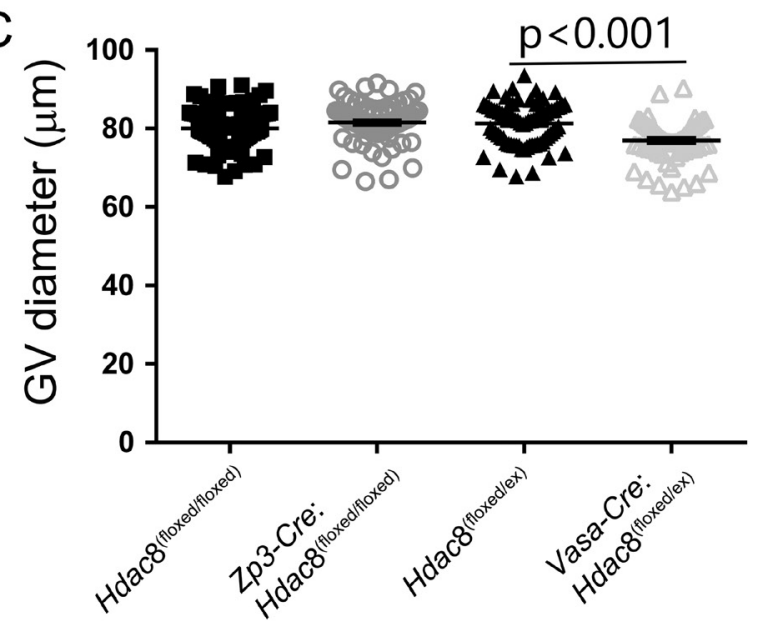

Figure 5 Vasa-Cre-mediated loss of oocyte-specific HDAC8 results in smaller oocytes. Transmitted light images of fully grown oocytes

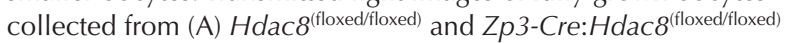
mice and (B) $H d a c 8^{\text {(floxed/ex) }}$ and Vasa-Cre:Hdac $8^{\text {(floxed/ex) }}$ mice. (C) Vasa-cre-mediated deletion of Hdac8 in oocytes affects oocyte diameter, $n=3$ mice for $H d a c 8^{\text {(floxed/floxed) }}(n=115$ oocytes), $n=3$ mice for Zp3-Cre:Hdac $8^{\text {(floxed/floxed) }}(n=76$ oocytes), $n=3$ mice for $H_{\text {dac }}{ }^{\text {(floxed/ex) }}(n=80$ oocytes) and $n=3$ mice for Vasa-

Cre:Hdac $8^{\text {(floxed/ex) }}(n=63$ oocytes). A student $t$-test was performed to examine statistical significance between genotypes and data are expressed as mean \pm S.E.M. $(P<0.001)$. Scale bar, $50 \mu \mathrm{m}$.

Hdac $8^{\text {(floxed/ex) }}(97.1 \%, n=42)$ and Vasa-Cre:Hdac $8^{\text {(floxed/ }}$ ex) $(96 \%, n=30)$ mice (Fig. 6A, B, C and D). These results demonstrate that loss of HDAC8 due to oocyte-specific Cre drivers does not affect $\mathrm{MI}$ spindle morphology and is therefore distinct from the RNAi-induced phenotype (Zhang et al. 2017). We performed chromosome spreads to assess the chromosome complement in mature metaphase II-arrested eggs following IVM (Fig. 6E). Using this method, we found that the percentage of euploid oocytes was the same across genotypes, with $85 \%$ of eggs
Table 1 Results of in vitro maturation to metaphase of meiosis I.

\begin{tabular}{|c|c|c|c|}
\hline \multicolumn{4}{|c|}{ No of oocytes (\%) } \\
\hline Genotype & Total & GV $(\%)$ & $\mathrm{MI}(\%)$ \\
\hline$H d a c 8^{\text {(floxed/floxed) }}$ & 141 & $12(8.5)$ & $129(91.5)$ \\
\hline Zp3-cre: $H d a c 8^{\text {(floxed/floxed) }}$ & 92 & $11(11.9)$ & $81(88.1)$ \\
\hline$H d a c 8^{\text {(floxed/ex) }}$ & 102 & $11(10.8)$ & $91(89.2)$ \\
\hline Vasa-cre: $H d a c 8^{\text {(floxed/ex) }}$ & 98 & $20(20.4)$ & $78(79.6)^{*}$ \\
\hline
\end{tabular}

Chi-squared, $* P<0.05$.

from mice of both genotypes being euploid (Hdac $8^{\text {(floxed/ }}$ ex), $n=101$ and Vasa-Cre:Hdac $\left.8^{\text {(floxed/ex) }}, n=46\right)$ (Fig. 6F).

\section{Role of HDAC8 in preimplantation embryo development}

To investigate whether the subfertility observed in Vasa-Cre:Hdac $8^{\text {(floxed/ex) }}$ mice was due to defects in preimplantation development, we isolated and cultured zygotes from Hdac $8^{\text {(floxed/ex) }}$ and Vasa-Cre:Hdac $8^{\text {(floxed/ex) }}$ mice and monitored their development up to the blastocyst stage. We observed a similar percentage of zygotes developing to blastocysts in the two genotypes, with $46 \%$ of zygotes from $H_{d a c} 8^{\text {(floxed/ex) }}$ $(n=24)$ developing to blastocyst compared to $59 \%$ in Vasa-Cre:Hdac $8^{\text {(floxed/ex) }}(n=39)$ mice (Table 2$)$. Thus the subfertility we observed in the Vasa-Cre:Hdac $8^{\text {(floxed/ex) }}$ mice is more likely attributable to subtle defects in oogenesis that result in smaller oocytes with compromised cytoplasmic competence rather than overt defects in chromosome segregation or preimplantation embryo development.

\section{Discussion}

Global deletion of HDAC8 in mice leads to perinatal lethality (Haberland et al. 2009). Here we report for the first time the phenotype of oocyte-specific knockouts of HDAC8 at both early (Vasa-Cre) and later stages (Zp3-Cre) of oocyte development. We reduced HDAC8 protein levels to $\sim 17 \%$ and $\sim 20 \%$ of control values using the Vasa-Cre and Zp3-Cre drivers, respectively. This reduction occurred without a compensatory upregulation of other class I HDACs (HDAC1, HDAC2 and HDAC3). Oocyte-specific loss of HDAC8 did not overtly affect folliculogenesis, providing further support that HDAC1 and HDAC2 have a more prominent role compared to HDAC8 during oocyte growth (Ma et al. 2012). However, we cannot exclude the possibility that changes occurred in the activity of the remaining class I HDACs nor can we rule out the possibility that the residual maternal HDAC8 in the oocyte is sufficient to maintain reproductive function in the knockout animals. While the Zp3-Cre driver was not associated with any fertility defect, the Vasa-Cre driver was associated with a mild subfertility phenotype. Since HDAC8 inhibitors are used to treat cancer, our findings suggest it will be 

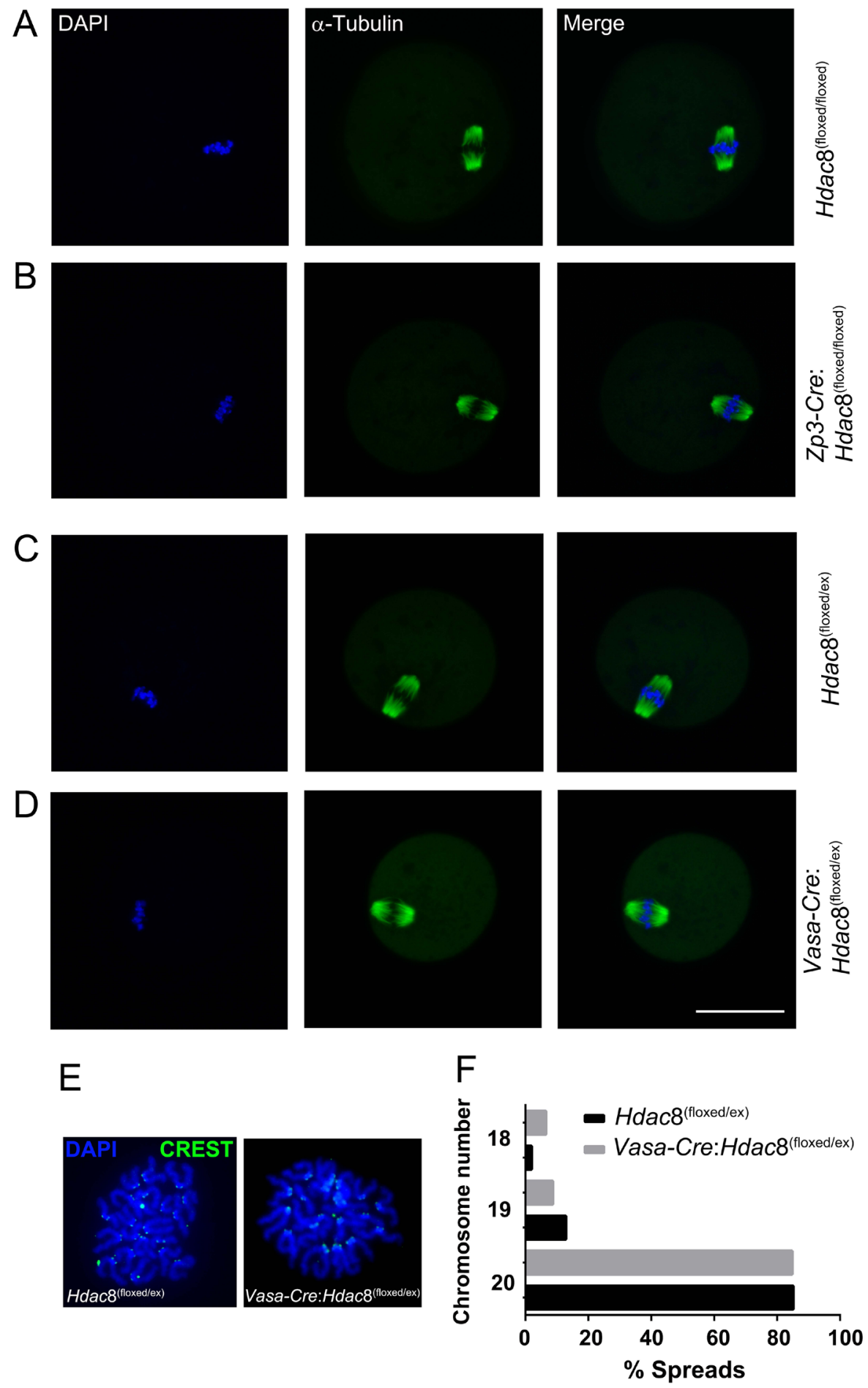

Figure 6 Oocyte-specific loss of HDAC8 does not affect spindle morphology or chromosome segregation. (A, B, C and D) Representative images of Ml oocytes from Hdac8 $8^{\text {(floxed/floxed) }}$ (A), Zp3-Cre:Hdac $8^{\text {(floxed/floxed }}(\mathrm{B}), H d a c 8^{\text {(floxed/ex) }}(\mathrm{C})$ and Vasa-Cre:Hdac ${ }^{\text {(floxed/ex) }}$ (D) mice showed normal spindle morphologies and chromosome alignment. Oocytes were immunostained with anti- $\alpha$-tubulin antibody to visualize spindles and

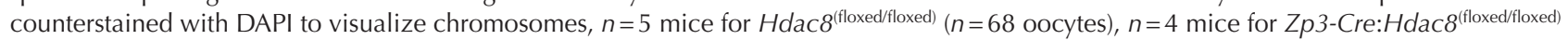
( $n=31$ oocytes), $n=4$ mice for Hdac $8^{\text {(floxed/ex) }}\left(n=42\right.$ oocytes) and $n=5$ mice for Vasa-Cre:Hdac $8^{\text {(floxed/ex) }}(n=30$ oocytes). The number of oocytes indicates those cells that contained $\mathrm{MI}$ spindles oriented parallel to the image plane which could be accurately evaluated for chromosome alignment. Scale bar, $50 \mu \mathrm{m}$. (E) Chromosome spreads from Hdac ${ }^{\text {(floxed/ex) }}\left(n=101\right.$ spreads from 8 mice) and Vasa-Cre:Hdac $8^{\text {(floxed/ex) }}(n=46$ spreads from 6 mice) mice showed normal chromosome number. Spreads were immunostained with anti-CREST antibody to visualize centromeres and counterstained with DAPI to visualize chromosomes. (F) Quantification of percentage of spreads with normal ( $n=20$ pairs of sister chromatids) and abnormal chromosome numbers. 
Table 2 Results of zygote culture.

\begin{tabular}{|c|c|c|c|c|c|}
\hline Genotype & Zygote & 2-cell $(\%)$ & 4-cell $(\%)$ & Morula (\%) & Blastocyst (\%) \\
\hline$H d a c 8^{\text {(floxed/ex) }}$ & 24 & $23(95.8)$ & $23(95.8)$ & $23(95.8)$ & $11(45.8)$ \\
\hline Vasa-cre: $H d a c 8^{\text {(floxed/ex) }}$ & 39 & $30(76.9)$ & $30(76.9)$ & $30(76.9)$ & $23(58.9)$ \\
\hline
\end{tabular}

Chi-squared, $P=0.21$.

worthwhile to determine whether these drugs affect posttreatment fertility.

Hdac8 is X-linked in humans and mouse, and its activity is independent of additional co-factors (Buggy et al. 2000, Hu et al. 2000, Van den Wyngaert et al. 2000, Chakrabarti et al. 2015). Interestingly histones are not bona fide substrates of HDAC8 but there is evidence that some histone acetylation increases after HDAC8 inhibitor treatment (Scholz et al. 2015). Whether the levels of acetylated histones increase in $\mathrm{Hdac} 8$ null oocytes requires further experimentation. Non-histone targets of HDAC8 include structural maintenance of chromosome 3 (SMC3), estrogen receptor $\alpha$ and cortical actin-binding protein, and p53 (Wilson et al. 2010, Deardorff et al. 2012, Wu et al. 2013, Olson et al. 2014). In fact, the role of HDAC8 in deacetylation of SMC3, a conserved subunit of the multimeric cohesin complex required for proper chromosome segregation, is wellcharacterized in yeast (Beckouet et al. 2010, Borges et al. 2010, Xiong et al. 2010, Li et al. 2017a) and in human (Deardorff et al. 2012). Mutations in HDAC8 have been reported to cause Cornelia de Lange Syndrome (CdLS) (Deardorff et al. 2012, Kaiser et al. 2014), and the affected individuals show severe growth as well as mental retardation. Similar to human, $\mathrm{Hdac} 8$ null mice exhibit growth retardation (Haberland et al. 2009). HDAC8 deacetylates SMC3, so that it will be available to regulate chromosome segregation during the following cell cycle (Beckouet et al. 2010, Borges et al. 2010, Xiong et al. 2010, Deardorff et al. 2012), suggesting that HDAC8 activity is important for recycling of SMC3. In oocytes, the existing literature suggests that there is no functional loading or exchange of cohesin subunits once oocytes are arrested in prophase I (Revenkova et al. 2010, Tachibana-Konwalski et al. 2010, Burkhardt et al. 2016), although the SMC3 subunit has not been specifically examined. If this hypothesis is true, then oocyte-specific knockout of Hdac8 using a Zp3-Cre driver should not cause a fertility defect. Our results are, therefore, consistent with the prevailing notion that there is no functional exchange of cohesin subunits and in particular, no recycling of SMC3.

Interestingly, when we knocked out oocyte-specific Hdac8 very early using the Vasa-Cre driver, we observed normal folliculogenesis but subfertility which may be due to defects in oocyte maturation. During our IVM experiment we did not observe any spindle or chromosome segregation defects, suggesting that even with loss of HDAC8, there is sufficient SMC3 available for sister chromatid cohesion and chromosome segregation.
The smaller oocyte size of Vasa-Cre:Hdac ${ }^{\text {(floxed/ex) }}$ mice indicates that some of these oocytes are not competent to undergo germinal vesicle breakdown. This suggests other HDAC8 target proteins, and deacetylation events may be necessary for proper oogenesis. It is worth mentioning that the Zp3-Cre driver will only affect oocytes whereas the Vasa-Cre driver removes Hdac8 in oocytes but all other tissues are heterozygous. Therefore we cannot rule out a contribution of heterozygosity in other tissues as an explanation for the subtle fertility defects with the Vasa-Cre driver.

Our findings contrast with experiments in which oocyte-specific knockdown of Hdac8 was performed in the fully grown oocyte with siRNAs against $\mathrm{Hdac} 8(70 \%$ loss of protein) or with an inhibitor against HDAC8 activity (Zhang et al. 2017). These acute treatments resulted in prominent kinetochore-microtubule attachment and spindle defects as well as aberrant chromosome alignment and aneuploidy. These effects would be predicted to have a profound negative impact on fertility, which we did not observe in our models, with the Zp3-Cre driver most closely resembling the timing of the knockdown in the previous work. However, the previous study was done using in vitro manipulations, and the authors did not rule out the possibility of off target or compensatory effects of their treatment regimes, which may account for the discrepancies between our findings.

In conclusion, we have used two different Cre mouse models, which express Cre in a temporal manner during oogenesis, to examine the role of HDAC8 in oogenesis and fertility. We provide evidence that HDAC8 is not required post-meiotic $S$ phase for oogenesis, fertility or preimplantation embryo development, but prior removal has negative consequences on GV diameter and maturation of oocytes. This may have downstream effects on blastocyst quality and/or implantation, but distinguishing these possibilities will require further experimentation.

\section{Supplementary data}

This is linked to the online version of the paper at https://doi.org/10.1530/REP-18-0560.

\section{Declaration of interest}

The authors declare that there is no conflict of interest that could be perceived as prejudicing the impartiality of the research reported. 


\section{Funding}

This work was supported by the Stowers Institute for Medical Research (J E G) and Northwestern University Department of Obstetrics and Gynecology Start Up Funds ( $F \quad E \quad D)$. Original data underlying this manuscript can be accessed from the Stowers Original Data Repository at https://www.stowers.org/research/publications/LIBPB-1371

\section{Acknowledgements}

The authors acknowledge Susmita Jasti for her work on preliminary experiments related to this study.

\section{References}

Beckouet F, Hu B, Roig MB, Sutani T, Komata M, Uluocak P, Katis VL, Shirahige K \& Nasmyth K 2010 An Smc3 acetylation cycle is essential for establishment of sister chromatid cohesion. Molecular Cell 39 689-699. (https://doi.org/10.1016/j.molcel.2010.08.008)

Bhaumik SR, Smith E \& Shilatifard A 2007 Covalent modifications of histones during development and disease pathogenesis. Nature Structural and Molecular Biology 14 1008-1016. (https://doi.org/10.1038/nsmb1337)

Bolden JE, Peart MJ \& Johnstone RW 2006 Anticancer activities of histone deacetylase inhibitors. Nature Reviews Drug Discovery 5 769-784. (https://doi.org/10.1038/nrd2133)

Borges V, Lehane C, Lopez-Serra L, Flynn H, Skehel M, Rolef Ben-Shahar T \& Uhlmann F 2010 Hos1 deacetylates Smc3 to close the cohesin acetylation cycle. Molecular Cell 39 677-688. (https://doi.org/10.1016/j. molcel.2010.08.009)

Bristol-Gould SK, Kreeger PK, Selkirk CG, Kilen SM, Cook RW, Kipp JL, Shea LD, Mayo KE \& Woodruff TK 2006 Postnatal regulation of germ cells by activin: the establishment of the initial follicle pool. Developmental Biology 298 132-148. (https://doi.org/10.1016/j. ydbio.2006.06.025)

Buggy JJ, Sideris ML, Mak P, Lorimer DD, McIntosh B \& Clark JM 2000 Cloning and characterization of a novel human histone deacetylase, HDAC8. Biochemical Journal 350 (Pt 1) 199-205. (https://doi. org/10.1042/bj3500199)

Burkhardt S, Borsos M, Szydlowska A, Godwin J, Williams SA, Cohen PE, Hirota T, Saitou M \& Tachibana-Konwalski K2016 Chromosome cohesion established by Rec8-Cohesin in fetal oocytes is maintained without detectable turnover in oocytes arrested for months in mice. Current Biology 26 678-685. (https://doi.org/10.1016/j.cub.2015.12.073)

Chakrabarti A, Oehme I, Witt O, Oliveira G, Sippl W, Romier C, Pierce RJ \& Jung M 2015 HDAC8: a multifaceted target for therapeutic interventions. Trends in Pharmacological Sciences 36 481-492. (https:// doi.org/10.1016/j.tips.2015.04.013)

Cordeiro MH, Kim SY, Ebbert K, Duncan FE, Ramalho-Santos J \& Woodruff TK 2015 Geography of follicle formation in the embryonic mouse ovary impacts activation pattern during the first wave of folliculogenesis. Biology of Reproduction 93 88. (https://doi.org/10.1095/ biolreprod.115.131227)

de Ruijter AJ, van Gennip AH, Caron HN, Kemp S \& van Kuilenburg AB 2003 Histone deacetylases (HDACs): characterization of the classical HDAC family. Biochemical Journal 370 737-749. (https://doi. org/10.1042/BJ20021321)

deVries WN, Binns LT, Fancher KS, Dean J, Moore R, Kemler R \& Knowles BB 2000 Expression of Cre recombinase in mouse oocytes: a means to study maternal effect genes. Genesis 26 110-112. (https://doi.org/10.1002/ (SICI)1526-968X(200002)26:2<110::AID-GENE2>3.0.CO;2-8)

Deardorff MA, Bando M, Nakato R, Watrin E, Itoh T, Minamino M, Saitoh K, Komata M, Katou Y, Clark D et al. 2012 HDAC8 mutations in Cornelia de Lange syndrome affect the cohesin acetylation cycle. Nature 489 313-317. (https://doi.org/10.1038/nature11316)

Duncan FE, Jasti S, Paulson A, Kelsh JM, Fegley B \& Gerton JL 2017 Age-associated dysregulation of protein metabolism in the mammalian oocyte. Aging Cell 16 1381-1393. (https://doi.org/10.1111/acel.12676)
Durst KL, Lutterbach B, Kummalue T, Friedman AD \& Hiebert SW 2003 The inv(16) fusion protein associates with corepressors via a smooth muscle myosin heavy-chain domain. Molecular and Cellular Biology 23 607-619. (https://doi.org/10.1128/MCB.23.2.607-619.2003)

Eichenlaub-Ritter U \& Peschke M 2002 Expression in in-vivo and in-vitro growing and maturing oocytes: focus on regulation of expression at the translational level. Human Reproduction Update 8 21-41. (https://doi. org/10.1093/humupd/8.1.21)

Eppig JJ \& Schroeder AC 1989 Capacity of mouse oocytes from preantral follicles to undergo embryogenesis and development to live young after growth, maturation, and fertilization in vitro. Biology of Reproduction $4 \mathbf{1}$ 268-276. (https://doi.org/10.1095/biolreprod41.2.268)

Gallardo T, Shirley L, John GB \& Castrillon DH 2007 Generation of a germ cell-specific mouse transgenic Cre line, vasa-Cre. Genesis 45 413-417. (https://doi.org/10.1002/dvg.20310)

Gosden R \& Lee B 2010 Portrait of an oocyte: our obscure origin. Journal of Clinical Investigation 120 973-983. (https://doi.org/10.1172/JCl41294)

Greer CB, Tanaka Y, Kim YJ, Xie P, Zhang MQ, Park IH \& Kim TH 2015 Histone deacetylases positively regulate transcription through the elongation machinery. Cell Reports 13 1444-1455. (https://doi. org/10.1016/j.celrep.2015.10.013)

Grunstein M 1997 Histone acetylation in chromatin structure and transcription. Nature 389 349-352. (https://doi.org/10.1038/38664)

Haberland M, Mokalled MH, Montgomery RL \& Olson EN 2009 Epigenetic control of skull morphogenesis by histone deacetylase 8. Genes and Development 23 1625-1630. (https://doi.org/10.1101/gad.1809209)

Hodges CA \& Hunt PA 2002 Simultaneous analysis of chromosomes and chromosome-associated proteins in mammalian oocytes and embryos. Chromosoma 111 165-169. (https://doi.org/10.1007/s00412-002-0195-3)

Hu E, Chen Z, Fredrickson T, Zhu Y, Kirkpatrick R, Zhang GF, Johanson K, Sung CM, Liu R \& Winkler J 2000 Cloning and characterization of a novel human class I histone deacetylase that functions as a transcription repressor. Journal of Biological Chemistry 275 15254-15264. (https:// doi.org/10.1074/jbc.M908988199)

Hubbert C, Guardiola A, Shao R, Kawaguchi Y, Ito A, Nixon A, Yoshida M, Wang XF \& Yao TP 2002 HDAC6 is a microtubule-associated deacetylase. Nature 417 455-458. (https://doi.org/10.1038/417455a)

Jenuwein T \& Allis CD 2001 Translating the histone code. Science 293 1074-1080. (https://doi.org/10.1126/science.1063127)

Kaiser FJ, Ansari M, Braunholz D, Concepcion Gil-Rodriguez $M$, Decroos C, Wilde JJ, Fincher CT, Kaur M, Bando M, Amor DJ et al. 2014 Loss-of-function HDAC8 mutations cause a phenotypic spectrum of Cornelia de Lange syndrome-like features, ocular hypertelorism, large fontanelle and X-linked inheritance. Human Molecular Genetics 23 2888-2900. (https://doi.org/10.1093/hmg/ddu002)

Lan ZJ, Xu X \& Cooney AJ 2004 Differential oocyte-specific expression of Cre recombinase activity in GDF-9-iCre, Zp3cre, and Msx2Cre transgenic mice. Biology of Reproduction 71 1469-1474. (https://doi. org/10.1095/biolreprod.104.031757)

Li S, Yue Z \& Tanaka TU 2017a Smc3 deacetylation by Hos1 facilitates efficient dissolution of sister chromatid cohesion during early anaphase. Molecular Cell 68 605.e4-614.e4. (https://doi.org/10.1016/j. molcel.2017.10.009)

Li X, Liu X, Gao M, Han L, Qiu D, Wang H, Xiong B, Sun SC, Liu H \& Gu L $2017 b$ HDAC3 promotes meiotic apparatus assembly in mouse oocytes by modulating tubulin acetylation. Development $144 \quad 3789-3797$. (https://doi.org/10.1242/dev.153353)

Luo J, Su F, Chen D, Shiloh A \& Gu W 2000 Deacetylation of p53 modulates its effect on cell growth and apoptosis. Nature 408 377-381. (https://doi. org/10.1038/35042612)

Ma P \& Schultz RM 2008 Histone deacetylase 1 (HDAC1) regulates histone acetylation, development, and gene expression in preimplantation mouse embryos. Developmental Biology 319 110-120. (https://doi. org/10.1016/j.ydbio.2008.04.011)

Ma P \& Schultz RM 2013 Histone deacetylase 2 (HDAC2) regulates chromosome segregation and kinetochore function via H4K16 deacetylation during oocyte maturation in mouse. PLOS Genetics $\mathbf{9}$ e1003377. (https://doi.org/10.1371/journal.pgen.1003377)

Ma P \& Schultz RM 2016 HDAC1 and HDAC2 in mouse oocytes and preimplantation embryos: specificity versus compensation. Cell Death and Differentiation 23 1119-1127. (https://doi.org/10.1038/ cdd.2016.31) 
Ma P, Pan H, Montgomery RL, Olson EN \& Schultz RM 2012 Compensatory functions of histone deacetylase 1 (HDAC1) and HDAC2 regulate transcription and apoptosis during mouse oocyte development. PNAS 109 E481-E489. (https://doi.org/10.1073/pnas.1118403109)

Martinez-Balbas MA, Bauer UM, Nielsen SJ, Brehm A \& Kouzarides T 2000 Regulation of E2F1 activity by acetylation. EMBO Journal 19 662-671. (https://doi.org/10.1093/emboj/19.4.662)

Minucci S \& Pelicci PG 2006 Histone deacetylase inhibitors and the promise of epigenetic (and more) treatments for cancer. Nature Reviews Cancer 6 38-51. (https://doi.org/10.1038/nrc1779)

Olson DE, Udeshi ND, Wolfson NA, Pitcairn CA, Sullivan ED, Jaffe JD, Svinkina T, Natoli T, Lu X, Paulk J et al. 2014 An unbiased approach to identify endogenous substrates of 'histone' deacetylase 8. ACS Chemical Biology 9 2210-2216. (https://doi.org/10.1021/cb500492r)

Pan H, O'brien MJ, Wigglesworth K, Eppig JJ \& Schultz RM 2005 Transcript profiling during mouse oocyte development and the effect of gonadotropin priming and development in vitro. Developmental Biology 286 493-506. (https://doi.org/10.1016/j.ydbio.2005.08.023)

Revenkova E, Herrmann K, Adelfalk C \& Jessberger R 2010 Oocyte cohesin expression restricted to predictyate stages provides full fertility and prevents aneuploidy. Current Biology 20 1529-1533. (https://doi. org/10.1016/j.cub.2010.08.024)

Scholz C, Weinert BT, Wagner SA, Beli P, Miyake Y, Qi J, Jensen LJ, Streicher W, McCarthy AR, Westwood NJ et al. 2015 Acetylation site specificities of lysine deacetylase inhibitors in human cells. Nature Biotechnology 33 415-423. (https://doi.org/10.1038/nbt.3130)

Sorensen RA \& Wassarman PM 1976 Relationship between growth and meiotic maturation of the mouse oocyte. Developmental Biology $\mathbf{5 0}$ 531-536. (https://doi.org/10.1016/0012-1606(76)90172-X)

Tachibana-Konwalski K, Godwin J, van der Weyden L, Champion L, Kudo NR, Adams DJ \& Nasmyth K 2010 Rec8-containing cohesin maintains bivalents without turnover during the growing phase of mouse oocytes. Genes and Development 24 2505-2516. (https://doi. org/10.1101/gad.605910)

Van den Wyngaert I, de Vries W, Kremer A, Neefs J, Verhasselt P, Luyten WH \& Kass SU 2000 Cloning and characterization of human histone deacetylase 8. FEBS Letters 478 77-83. (https://doi.org/10.1016/ S0014-5793(00)01813-5)

Wickramasinghe D \& Albertini DF 1992 Centrosome phosphorylation and the developmental expression of meiotic competence in mouse oocytes. Developmental Biology 152 62-74. (https://doi.org/10.1016/00121606(92)90156-B)

Wickramasinghe D, Ebert KM \& Albertini DF 1991 Meiotic competence acquisition is associated with the appearance of $\mathrm{M}$-phase characteristics in growing mouse oocytes. Developmental Biology 143 162-172. (https://doi.org/10.1016/0012-1606(91)90063-9)

Wilson BJ, Tremblay AM, Deblois G, Sylvain-Drolet G \& Giguere V 2010 An acetylation switch modulates the transcriptional activity of estrogenrelated receptor alpha. Molecular Endocrinology 24 1349-1358. (https:// doi.org/10.1210/me.2009-0441)

Wu J, Du C, Lv Z, Ding C, Cheng J, Xie H, Zhou L \& Zheng S 2013 The upregulation of histone deacetylase 8 promotes proliferation and inhibits apoptosis in hepatocellular carcinoma. Digestive Diseases and Sciences 58 3545-3553. (https://doi.org/10.1007/s10620-013-2867-7)

Xiong B, Lu S \& Gerton JL 2010 Hos1 is a lysine deacetylase for the Smc3 subunit of cohesin. Current Biology 20 1660-1665. (https://doi. org/10.1016/j.cub.2010.08.019)

Zhang K, Lu Y, Jiang C, Liu W, Shu J, Chen X, Shi Y, Wang E, Wang L, Hu Q et al. 2017 HDAC8 functions in spindle assembly during mouse oocyte meiosis. Oncotarget 8 20092-20102. (https://doi.org/10.18632/ oncotarget.15383)

Received 30 October 2018

First decision 19 November 2018

Revised manuscript received 19 December 2018

Accepted 8 January 2019 